\title{
Landmarks as Navigation-Aids for Multiple Robots
}

\author{
Bibhya Sharma, Avinesh Prasad, Ritesh Chand, Sandeep Kumar, Jai Raj and Jito Vanualailai
}

\begin{abstract}
The paper presents selected landmarks as navigation-aids or waypoints for multiple car-like robots in a contained workspace cluttered with randomly fixed obstacles and landmarks. A new metrics is designed to select specific landmarks (which are treated as waypoints) falling in the robots' field of view and with a minimum distance away from each other and their targets. A new metric is also defined to obtain the robot's field of view at every iteration. Using the Lyapunov-based control scheme (LbCS) nonlinear acceleration-based stabilizing control laws are derived for navigation amongst obstacles and landmarks en route the final destination via selected landmarks or waypoints. The proposed technique and the new control laws are verified via interesting computer simulations.
\end{abstract}

Keywords: Motion control, landmark navigation, obstacle avoidance, car-like robot, stability.

\section{INTRODUCTION}

Planning of feasible trajectories and control of robotic systems have been active area of research for past decades. Nowadays, the wheeled robots (vehicles) are playing vital roles in human livelihood and endeavours, and found in most industries that require automation and repetition. Under this category the robots with nonholonomic constraints have been researched and utilized more although their nonholonomic constraints make the problem quite demanding and challenging [1]. Major applications such as sea and space exploration, surveillance, rescue missions, pursuit-evasion, surveying, pedestrian navigation, waste management, foraging and multi-robot reconnaissance utilises such robotic systems [2],[3], [4], [5]. For surveillance and exploration purposes mostly, the presence of cues (natural or artificial) landmarks will be of an advantage as the robot can move from a destination to another destination easily with the added guidance. In the field of robotics, a significant amount of work has been carried out with landmarks for navigation of autonomous robots. Landmarks are mainly used to guide the robot to a desired goal [6], [7], [8], [9], [10], [7], and to allow the robots to determine their position with respect to the landmarks (mapping and localization) ([11], [12], [13]).

Bibhya Sharma is an Associate Professor at the School of Computing, Information and Mathematical Sciences, University of the South Pacific (email: sharma_b@usp.ac.fj).

Avinesh Prasad is a Senior Lecturer at the School of Computing, Information and Mathematical Sciences, University of the South Pacific (e-mail: prasad_ai@usp.ac.fj).

Ritesh Chand is a Teaching Assistant at the School of Computing, Information and Mathematical Sciences, University of the South Pacific (e-mail: chand_rd@usp.ac.fj).

Sandeep Kumar is an Assistant Lecturer at the School of Computing, Information and Mathematical Sciences, University of the South Pacific (e-mail: sandeep.a.kumar@usp.ac.fj).

Jai Raj is a PhD student at University of the South Pacific (e-mail: raj j@usp.ac.fj).

Jito Vanualailai is a Professor at the School of Computing, Information and Mathematical Sciences, University of the South Pacific (e-mail: vanualailai@usp.ac.fj).
In literature, the concept of waypoints is linked to landmarks where the waypoints can be those landmarks selected for a specific task. In addition, waypoints can also include physical objects, devices or coordinates that hold navigation details for robot navigation or human movements [6], [14].

Landmarks that already exist naturally in the environment and may have purpose outside of robot navigation are known as natural landmarks, whereas special features or objects designed and added to the environment are known as artificial landmarks. The seminal purpose of artificial landmarks is to enable autonomous robot navigation [15]. For instance, beacon floaters are being particularly used to guide dispatches securely into wharf field. Together with this, street signs, activity lights and passerby intersections are additionally utilised as historic points for route purposes.

The issue of discovering an ideal arrangement of landmarks positions has been tended to from a few perspectives in the past [7], [8], [9],[11], [12], [13]. Work that has being carried out in the theoretical framework of landmarks in the recent past has been contained mainly to selection, detection and learning of the landmarks. This paper will extend the obstacle avoidance schemes together with path planning of car-like robots developed before in [16], [17], [3], by the utilisation of landmarks in the workspace which will aid the robots to navigate to their respective targets. The literature on landmarks for navigation focusses on mapping and localization which is basically determining the position of the robot and selecting the best route for the robot to reach its goal autonomously [7]. However, there is growing interest in utilizing landmarks as navigation-aids for robots in known/unknown environments where the landmarks act as active waypoints. We will consider such a complex workspace which will consist of multiple landmarks (treated as waypoints) and obstacles. However, factors such as cost and time make navigation via all landmarks unimaginative, impractical and tedious [18]. Hence, in this paper, the car-like robots will select a set of landmarks from all scattered landmarks in the workspace via which each robot can explore the workspace and converge to its target. The stability of the system will be analysed using the Direct Method of Lyapunov. Simulations will be used to show that the extracted controllers guarantee, for some initial conditions, point stabilities.

The nonlinear controllers have been incorporated in the kinodynamic model by means of a energy like Lyapunov function or total potentials developed from the artificial potential field method known as the Lyapunov-based Control Scheme (LbCS). This method offers a greater degree of flexibility in taking into account all the system and dynamic constraints, boundary restrictions, waypoints and obstacles within the robotic workspace and finally attaining the desired goal [16], [17], [3].

This paper is organized as follows: In Section II, the kinodynamic model of a car-like robot is detailed. Section III 
defines landmarks, field of view and target and gives the landmark changing rule and its attraction function. In Section IV, we include various types of obstacles and their repulsive functions. A tentative Lyapunov function is proposed in Section V from which the control laws are extracted. Section VII looks at a simulation result. Finally, in Section VIII the paper concludes with a discussion on its contributions and potential future work.

\section{SySTEM MODELLING}

We consider $n$ rear wheel driven car-like robots, the ODEs governing their motion adopted from [3]. As seen in Figure 1, the $\left(x_{i}, y_{i}\right)$ denotes the center of mass $(\mathrm{CoM})$ of the $i$ th carlike robot, its orientation with respect to the $z_{1}$-axis is given by $\theta_{i}$, while its steering angle with respect to the longitudinal axis is given as $\phi_{i}$ gives. The $L$ is the distance between two axles, while $l$ the length of each axle. The configuration of the $i$ th car is given as $\left(x_{i}, y_{i}, \theta_{i}, \phi_{i}\right) \in \mathbb{R}^{4}$, and its position is given as the point $\left(x_{i}, y_{i}\right) \in \mathbb{R}^{2}$. The kinodynamic model of

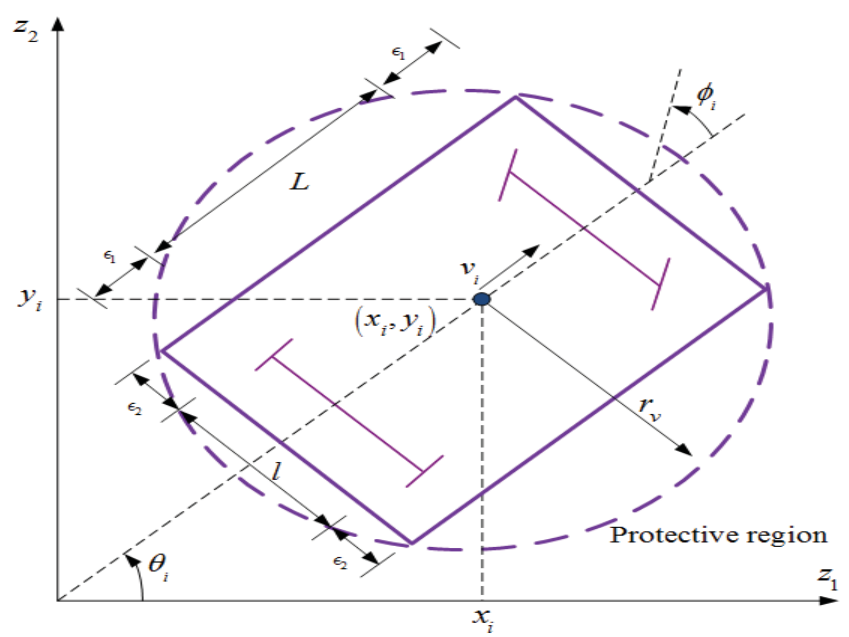

Fig. 1. A rear wheel driven vehicle with front wheel steering and steering angle $\phi_{i}$.

the $i$ th car-like robot is given as (adopted from [3]):

$$
\left.\begin{array}{l}
\dot{x}_{i}=v_{i} \cos \theta_{i}-\frac{L}{2} \omega_{i} \sin \theta_{i}, \\
\dot{y}_{i}=v_{i} \sin \theta_{i}+\frac{L}{2} \omega_{i} \cos \theta_{i}, \\
\dot{\theta}_{i}=\omega_{i}, \quad \dot{v}_{i}=\sigma_{i 1}, \quad \dot{\omega}_{i}=\sigma_{i 2},
\end{array}\right\}
$$

where $v_{i}$ and $\omega_{i}$ are the translational and rotational velocities, and $\sigma_{i 1}$ and $\sigma_{i 2}$ are the translational and rotational accelerations. $\sigma_{i 1}$ and $\sigma_{i 2}$ are are known as the controllers of the robotic system. We assume in the model that there is no slippage, that is, $\dot{x}_{i r} \sin \theta_{i}-\dot{y}_{i r} \cos \theta_{i}=0$ and $\dot{x}_{i f} \sin \left(\theta_{i}+\phi_{i}\right)-\dot{y}_{i f} \cos \left(\theta_{i}+\phi_{i}\right)=0$ and pure rolling, that is, $\dot{x}_{i} \cos \theta_{i}+\dot{y}_{i} \sin \theta_{i}=v_{i}$ where $\left(x_{i r}, y_{i r}\right)$ and $\left(x_{i f}, y_{i f}\right)$ are the coordinates of the centers of the rear and front axles, respectively. Hereafter, the vector notation $\mathbf{x}_{i}=\left(x_{i}, y_{i}, \theta_{i}, v_{i}, \omega_{i}\right) \in$ $\mathbb{R}^{5}$ will be used to describe the variables in (1). Without loss of generality, we let $\mathbf{x}=\left(\mathbf{x}_{1}, \mathbf{x}_{2}, \ldots, \mathbf{x}_{n}\right) \in \mathbb{R}^{5 n}$.

The principal objective of the research paper is to design new controllers $\sigma_{i 1}$ and $\sigma_{i 2}$ so that the $i$ th car-like robots can maneuver through a set of landmarks and obstacles and finally reach the designated targets. We adopt the following definition of a circular target from [16]:

Definition 1: The target for the $i$ th vehicle is a disk of center $\left(p_{i 1}, p_{i 2}\right)$ and radius $r_{i T}$ which is described as the set

$$
T_{i}=\left\{\left(z_{1}, z_{2}\right) \in \mathbb{R}^{2}:\left(z_{1}-p_{i 1}\right)^{2}+\left(z_{2}-p_{i 2}\right)^{2} \leq r_{i T}^{2}\right\} .
$$

\section{LANDMARKS}

Definition 2: The $k$ th landmark with the rectangular position $\left(l x_{k}, l y_{k}\right)$ in the $z_{1} z_{2}$-plane is given by

$$
L M_{k}=\left\{\left(z_{1}, z_{2}\right) \in \mathbb{R}^{2}:\left(z_{1}-l x_{k}\right)^{2}+\left(z_{2}-l y_{k}\right)^{2}=0\right\}
$$

for $k=1,2, \ldots, r$.

Assumtion 1: The position of the landmarks are priori known.

Assumtion 2: The vehicle goes through each of the selected landmarks (waypoints) and finally converges to the center of the predetermined target.

We further denote

$$
d_{i k}(t)=\sqrt{\left(x_{i}(t)-l x_{k}\right)^{2}+\left(y_{i}(t)-l y_{k}\right)^{2}},
$$

for $k=1,2, \ldots, r$ as the distance between the center of the $i$ th car-like robot and the $k$ th landmark.

\section{A. Selection of Landmarks}

A workspace may contain many landmarks; however, factors such as cost and time make navigation via all landmarks unimaginative, impractical and tedious [18]. Instead only those landmarks will be selected and navigated that lie in a given field of view of the robot and also only existing between the robot's initial and target positions. The field of view is defined as follows:

Definition 3: Given a predetermined scalar $\zeta \in(0, \pi)$, the set

$$
\begin{aligned}
F V_{i}= & \left\{\left(z_{1}, z_{2}\right) \in \mathbb{R}^{2}: \mid \operatorname{atan} 2\left(z_{2}-y_{i}, z_{1}-x_{i}\right)-\operatorname{atan} 2\left(p_{i 2}\right.\right. \\
& \left.-y_{i}, p_{i 1}-x_{i}\right)|<\zeta \cup 2 \pi-| \operatorname{atan} 2\left(z_{2}-y_{i}, z_{1}-x_{i}\right) \\
& \left.-\operatorname{atan} 2\left(p_{i 2}-y_{i}, p_{i 1}-x_{i}\right) \mid<\zeta\right\}
\end{aligned}
$$

is the $i$ th robot's field of view.

We define a metric for the selection of the landmarks as follows:

1) The selected landmark should lie within the $F V_{i}$.

2) The selected landmark should be sufficiently away from the target.

3) The selected landmark should lie between the robot's initial $\left(x_{i}, y_{i}\right)$ and their target $\left(p_{i 1}, p_{i 2}\right)$ positions.

4) No two selected landmarks should be close to each other (minimum distance between landmarks defined by user).

We now develop an algorithm for the selection of landmark based on the above metric. Let $S=\{1,2,3, \ldots, r\}$ such that 
$d_{i 1}<d_{i 2}<\cdots<d_{i r}$ and define the $k$ th subsets, $S_{i k} \subseteq S$, for $k=1,2, \ldots, r$, as

$$
S_{i k}= \begin{cases}\{k\}, & \text { if }\left(l x_{k}, l y_{k}\right) \in F V_{i}, d_{i k}>D^{*} \text { and } \\
\{\}, & \begin{array}{l}
\left\|\left(x_{k}-p_{i 1}, l y_{k}-p_{i 2}\right)\right\|>D^{*} \\
\text { otherwise }
\end{array}\end{cases}
$$

where $D^{*}>0$ is the minimum distance between any two selected landmarks. Then consider set $M_{i}$, a subset of $S$, as

$$
M_{i}=\bigcup_{k=1}^{r} S_{i k} \subseteq S .
$$

We can easily see that the set $M_{i}$ will contain only those values of $k$ whose landmarks lie in $F V_{i}$. Letting $s_{i}=\left|M_{i}\right|$ be the cardinality of the set $M_{i}, M_{i}$ contains a total of $s_{i} \leq r$ values in ascending order. Let $M_{i}[u]$ be the $u$ th entry of $\bar{M}_{i}$.

\section{B. Navigation via the Selected Landmarks}

For the $i$ th vehicle to navigate through the selected landmarks, the following landmark attraction potential function is considered which will appear in a Lyapunov function to be proposed in Section V:

$V_{i u}(\mathbf{x})=\frac{1}{2} \rho_{i u}\left[\left(x_{i}-l x_{M_{i}[u]}\right)^{2}+\left(y_{i}-l y_{M_{i}[u]}\right)^{2}+v_{i}^{2}+\omega_{i}^{2}\right]$

for $u=1,2, \ldots, s_{i}+1$. This function is a measure of the distance between the center of the vehicle and the $u$ th landmark. Note that $\rho_{i u}$ is an updating rule for changing of landmarks and is obtained from the pseudo-code below:

$$
\begin{aligned}
& \left(l x_{M_{i}\left[s_{i}\right]+1}, l y_{M_{i}\left[s_{i}\right]+1}\right):=\left(p_{i 1}, p_{i 2}\right) \text {; } \\
& d_{i M_{i}[0]}=\left\|\left(x_{i}(t), y_{i}(t)\right)-\left(x_{i}(0), y_{i}(0)\right)\right\| \text {; } \\
& \text { for } v=1 \text { to } s_{i} \\
& \text { if } d_{i M_{i}[v-1]}=0 \\
& \text { for } u=1 \text { to } s_{i} \\
& \text { if } u=v \\
& \rho_{i u}=1 \text {; } \\
& \text { else } \rho_{i u}=0 \text {; } \\
& \text { end } \\
& \text { end } \\
& \text { end } \\
& \text { end } \\
& \text { end }
\end{aligned}
$$

\section{AVOIdAnCE of OBstacles}

In this section, we consider possible forms of obstacles the car-like robots may encounter en route their targets. These include workspace restrictions, fixed obstacles and dynamic constraints. Also for the entire body of a robot to safely steer past an obstacle, we enclose it by the smallest circle possible. Given the clearance parameters $\epsilon_{1}$ and $\epsilon_{2}$, we can enclose the robot in a circular protective region which is centered at $\left(x_{i}, y_{i}\right)$ and has radius of $r_{V}=\frac{1}{2} \sqrt{\left(L+2 \epsilon_{1}\right)^{2}+\left(l+2 \epsilon_{2}\right)^{2}}$.

\section{A. Workspace Restrictions}

We want the car-like robots to operate in a closed workspace. We therefore adopt the following definition of a rectangular workspace from [3].
Definition 4: The workspace $W S$ is a fixed, closed and bounded rectangular region which is precisely the set

$$
W S=\left\{\left(z_{1}, z_{2}\right) \in \mathbb{R}^{2}: 0 \leq z_{1} \leq \eta_{1}, 0 \leq z_{2} \leq \eta_{2}\right\} .
$$

These boundaries will be considered as fixed obstacles, and they have to be avoided by the robots. For avoidance, we shall adopt the following obstacle avoidance functions from [16], for the left, right, lower and upper boundaries, respectively:

$$
\begin{array}{ll}
W_{i 1}(\mathbf{x})=x_{i}-r_{V}, & W_{i 2}(\mathbf{x})=\eta_{1}-\left(r_{V}+x_{i}\right), \\
W_{i 3}(\mathbf{x})=y_{i}-r_{V}, & W_{i 4}(\mathbf{x})=\eta_{2}-\left(r_{V}+y_{i}\right) .
\end{array}
$$

\section{B. Moving Obstacles}

We note that the $j$ th robot becomes a moving obstacle for the $i$ th robot. Hence, for the inter-robot avoidance, we consider the potential function

$$
M O_{i j}(\mathbf{x})=\frac{1}{2}\left[\left(x_{i}-x_{j}\right)^{2}+\left(y_{i}-y_{j}\right)^{2}-2 r_{V}^{2}\right]
$$

for $j \neq i$.

\section{Fixed Elliptic Obstacles}

We fix $q>0$ elliptic obstacles in workspace $W S$. The (2) th obstacle (for $l=1,2, \ldots, q$ ) is assumed to be an ellipse centered at $\left(o_{l 1}, o_{l 2}\right)$ and is defined as the set

$$
\begin{aligned}
O_{l}:=\left\{\left(z_{1}, z_{2}\right)\right. & \in \mathbb{R}^{2}: \frac{\left[\left(z_{1}-o_{l 1}\right) \cos \varphi_{l}+\left(z_{2}-o_{l 2}\right) \sin \varphi_{l}\right]^{2}}{a_{l}^{2}} \\
+ & \left.\frac{\left[\left(z_{2}-o_{l 2}\right) \cos \varphi_{l}-\left(z_{1}-o_{l 1}\right) \sin \varphi_{l}\right]^{2}}{b_{l}^{2}} \leq 1\right\},
\end{aligned}
$$

for some constants $a_{l}, b_{l}$ and $\varphi_{l}$. For its avoidance we consider

$$
\begin{aligned}
F O_{i l}(\mathbf{x}) & =\frac{1}{2}\left[\frac{\left[\left(x_{i}-o_{l 1}\right) \cos \varphi_{l}+\left(y_{i}-o_{l 2}\right) \sin \varphi_{l}\right]^{2}}{\left(r_{V}+a_{l}\right)^{2}}\right. \\
& \left.+\frac{\left[\left(y_{i}-o_{l 2}\right) \cos \varphi_{l}-\left(x_{i}-o_{l 1}\right) \sin \varphi_{l}\right]^{2}}{\left(r_{V}+b_{l}\right)^{2}}-1\right]
\end{aligned}
$$

\section{Unselected Landmarks as Fixed Obstacles}

For simplicity, The landmarks which do not lie in the field of view of the robots are not considered in the avoidance scheme in this research. The robots will not encounter them during their journey to their targets. However, those landmarks that lie within $F V_{i}$ but were not selected due to the rules of the metrics are considered here as point obstacles, which the robots must avoid. Define the $k$ th subsets, $S_{i k}^{*} \subseteq S$, for $k=1,2, \ldots, r$, as

$$
S_{i k}^{*}= \begin{cases}\{k\}, & \text { if }\left(l x_{k}, l y_{k}\right) \in F V_{i} \text { and } d_{i k}<D^{*} \\ \{\}, & \text { or }\left\|\left(l x_{k}-p_{i 1}, l y_{k}-p_{i 2}\right)\right\|<D^{*} \\ \{\text { otherwise }\end{cases}
$$

Note that the the subset $S_{i k}^{*}$ will be calculated at $t=0$ and will be updated when the robot reaches each selected landmark. we now define set $M_{i}^{*}$, also a subset of $S$, as follows:

$$
M_{i}^{*}=\bigcup_{k=1}^{r} S_{i k}^{*} \subseteq S \text {. }
$$

Set $M_{i}^{*}$ will include the unselected landmarks that lie in $F V_{i}$ but are within the distance $D^{*}$ from the previous landmark or 
within the distance $D^{*}$ from the target. Say that $M_{i}^{*}$ contains a total of $s_{i}^{*} \leq r$ values in ascending order and we let $M_{i}^{*}[v]$ be the $v$ th entry of $M_{i}^{*}$. For the robot to avoid these unselected landmarks, we consider the potential function

$$
H_{i v}(\mathbf{x})=\frac{1}{2}\left[\left(x_{i}-l x_{M_{i}^{*}[v]}\right)^{2}+\left(y_{i}-l y_{M_{i}^{*}[v]}\right)^{2}-r_{V}^{2}\right]
$$

for $v=1,2, \ldots, s_{i}^{*}$.

\section{E. Artificial Obstacles}

The instantaneous velocities of the vehicle are restricted due to safety considerations. Firstly, we impose a maximum velocity $v_{\max }$ of the robot to operate within, and secondly, the steering angle of the front wheel is also bounded. In accordance with LbCS, we construct artificial obstacles for these constraints:

$$
\begin{aligned}
& A O_{i 1}=\left\{v_{i} \in \mathbb{R}: v_{i} \leq-v_{\max } \text { or } v_{i} \geq v_{\max }\right\}, \\
& A O_{i 2}=\left\{\omega \in \mathbb{R}: \omega_{i} \leq-v_{\max } /\left|\rho_{\min }\right| \text { or } \omega_{i} \geq v_{\max } /\left|\rho_{\min }\right|\right\},
\end{aligned}
$$

where $\rho_{\min }=\ell_{1} / \tan \left(\phi_{\max }\right), \phi_{\max }$ is the maximal steering angle. The following functions are adopted for the avoidance of the aforementioned artificial obstacles:

$$
\begin{array}{r}
U_{i 1}(\mathbf{x})=\frac{1}{2}\left(v_{\max }^{2}-v_{i}^{2}\right), \\
U_{i 2}(\mathbf{x})=\frac{1}{2}\left(\frac{v_{\max }^{2}}{\rho_{\min }^{2}}-\omega_{i}^{2}\right) .
\end{array}
$$

\section{The Lyapunov Function And Controller Design}

We will utilize the LbCS to derive acceleration-based controllers of the group of car-like robots. The total potentials will consist of attractive potential field functions for target attraction and repulsive potential field functions for obstacle avoidance and obeying system constraints and limitations. while the distance-based avoidance functions are treated as attractive potential field functions, the repulsive potential field functions are rational functions constructed using positive tuning parameters populating the numerator with obstacle avoidance functions encoded in the denominators. An example of total potentials is illustration via a $3 \mathrm{D}$ visualization in Figure 2 and a contour plot in Figure 3.

\section{A. Auxiliary Function}

To make sure of the fact that the controllers of the carlike robots vanish at their targets, we design a new potential function and multiply it to the inverse of each avoidance functions designed above:

$$
F_{i u}(\mathbf{x})=\frac{1}{2} \rho_{i u}\left[\left(x_{i}-l x_{M_{i}[u]}\right)^{2}+\left(y_{i}-l y_{M_{i}[u]}\right)^{2}\right]
$$

for $u=1,2, \ldots, s_{i}+1$ as an auxiliary function.

\section{B. Lyapunov Candidate Function}

Combining all the attractive and avoidance functions and introducing control parameters, $\alpha_{i m}>0, \beta_{i j}>0, \gamma_{i l}>0$,

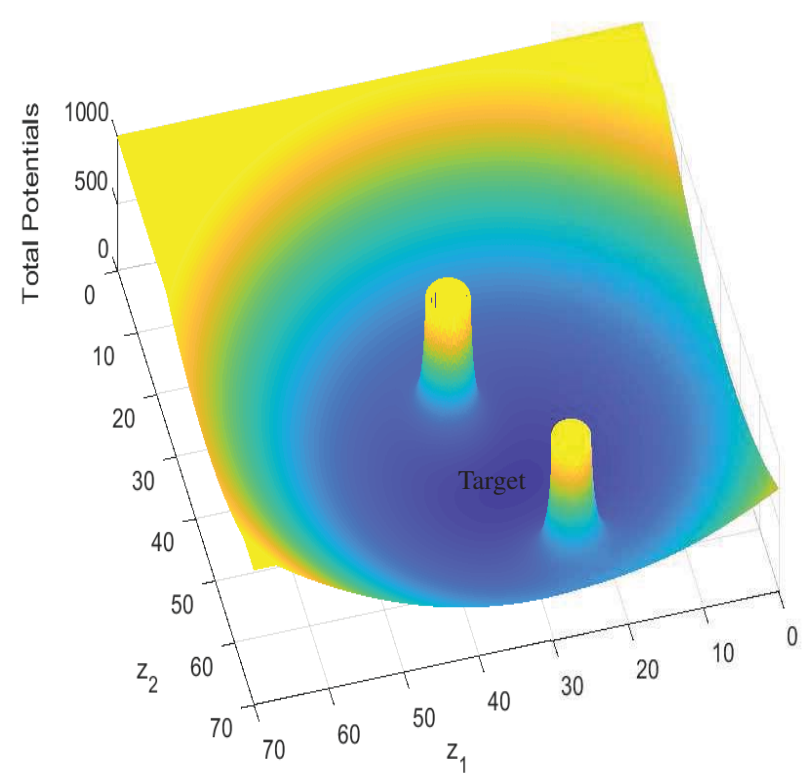

Fig. 2. 3D Visualization

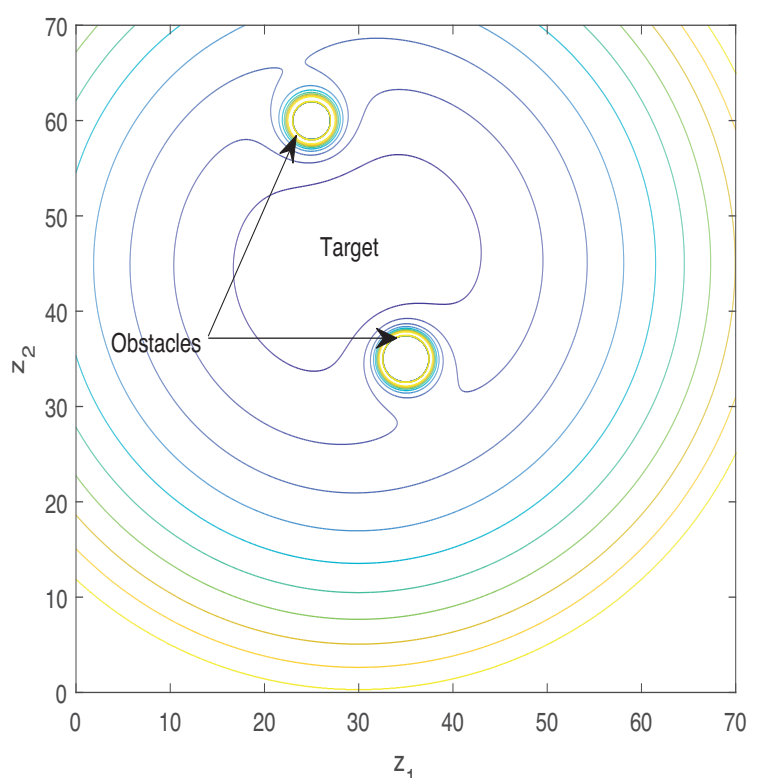

Fig. 3. Contour Plot: The total potentials generated for target attraction and obstacle avoidance. The target is located at $(30,45)$ with radius of 1 and the obstacles are fixed at $(25,60)$ and $(35,35)$ with radius of 2 and $\sqrt{ } 6$ respectively. 
function for system (1) is defined as

$$
\begin{aligned}
L(\mathbf{x})= & \sum_{i=1}^{n} \sum_{u=1}^{s_{i}+1}\left[V_{i u}(\mathbf{x})+F_{i u}(\mathbf{x})\left(\sum_{m=1}^{4} \frac{\alpha_{i m}}{W_{i m}(\mathbf{x})}\right.\right. \\
& +\sum_{\substack{j=1 \\
j \neq i}}^{n} \frac{\beta_{i j}}{M O_{i j}(\mathbf{x})}+\sum_{l=1}^{q} \frac{\gamma_{i l}}{F O_{i l}(\mathbf{x})} \\
& \left.\left.+\sum_{v=1}^{s_{i}^{*}} \frac{\xi_{i v}}{H_{i v}(\mathbf{x})}+\sum_{p=1}^{2} \frac{\kappa_{i p}}{U_{i p}(\mathbf{x})}\right)\right]
\end{aligned}
$$

The Lyapunov function $L(\mathbf{x})$, is defined, piecewise continuous and positive over the domain

$$
\begin{aligned}
D=\{\mathbf{x} & \in \mathbb{R}^{5 n}: W_{i m}(\mathbf{x})>0, m=1, \ldots, 4 \\
& M O_{i j}(\mathbf{x})>0, i, j=1, \ldots, n, j \neq i \\
& F O_{i l}(\mathbf{x})>0, l=1, \ldots, q ; U_{i p}(\mathbf{x})>0, p=1,2 \\
& \left.H_{i v}(\mathbf{x})>0, v=1, \ldots, s_{i}^{*}\right\} \backslash \bigcup_{i=1}^{n} \bigcup_{u=1}^{s_{i}} L M_{M_{i}[u]} .
\end{aligned}
$$

\section{Controller Design}

The various components of $L(\mathbf{x})$ are differentiated separately with respect to $t$ to extract the nonlinear controllers for system (1)

$$
\begin{gathered}
\dot{L}(\mathbf{x})=\sum_{i=1}^{n}\left\{\left[f_{i 1} \cos \theta_{i}+f_{i 2} \sin \theta_{i}+f_{i 3} \sigma_{i 1}\right] v_{i}\right. \\
\left.+\left[\frac{L}{2} f_{i 2} \cos \theta_{i}-\frac{L}{2} f_{i 1} \sin \theta_{i}+f_{i 4} \sigma_{i 2}\right] \omega_{i}\right\} .
\end{gathered}
$$

where the functions $f_{1}$ to $f_{4}$ are defined as

$$
\begin{aligned}
& f_{i 1}(\mathbf{x})=\sum_{i=1}^{n} \sum_{u=1}^{s_{i}+1} \rho_{i_{M_{i}[u]}}\left[\left(1+\sum_{m=1}^{4} \frac{\alpha_{i m}}{W_{i m}(\mathbf{x})}\right)\left(x_{i}-l x_{M_{i}[u]}\right)\right. \\
& +\left(\sum_{v=1}^{s_{i}^{*}} \frac{\xi_{i v}}{H_{i v}(\mathbf{x})}+\sum_{l=1}^{q} \frac{\gamma_{i l}}{F O_{i l}(\mathbf{x})}+\sum_{p=1}^{2} \frac{\kappa_{i p}}{U_{i p}}\right)\left(x_{i}-l x_{M_{i}[u]}\right) \\
& +\left(\sum_{\substack{j=1 \\
j \neq i}}^{n} F_{j M_{i}[u]}(\mathbf{x})\left(\frac{\beta_{i l}}{M O_{i j}}+\frac{\beta_{i l}}{M O_{j i}}\right)\right)\left(x_{i}-l x_{M_{i}[u]}\right) \\
& -\sum_{\substack{j=1 \\
j \neq i}}^{n} F_{i M_{i}[u]}(\mathbf{x}) F_{j M_{i}[u]}(\mathbf{x})\left(\frac{\beta_{i j}\left(x_{i}-x_{j}\right)}{M O_{i j}^{2}}-\frac{\beta_{j i}\left(x_{j}-x_{i}\right)}{M O_{j i}^{2}}\right) \\
& -F_{i M_{i}[u]}\left(\frac{\alpha_{i 1}}{W_{i 1}^{2}}-\frac{\alpha_{i 2}}{W_{i 2}^{2}}+\sum_{v=1}^{s_{i}^{*}} \frac{\xi_{i v}}{H_{i v}^{2}(\mathbf{x})}\left(x_{i}-l x_{M^{*}{ }_{i}[v]}\right)\right) \\
& +\left(F_{i M_{i}[u]} \sum_{l=1}^{q} \frac{\gamma_{i l} \sin \psi_{l}\left(\left(y_{i}-o_{l 2}\right) \cos \psi_{l}+\left(x_{i}-o_{l 1}\right) \sin \psi_{l}\right)}{F O_{i l}^{2}(\mathbf{x})\left(r_{i v}+b_{l}\right)^{2}}\right) \\
& \left.-\left(F_{i M_{i}[u]} \sum_{l=1}^{q} \frac{\gamma_{i l} \cos \psi_{l}\left(\left(x_{i}-o_{l 1}\right) \cos \psi_{l}+\left(y_{i}-o_{l 2}\right) \sin \psi_{l}\right)}{F O_{i l}^{2}(\mathbf{x})\left(r_{v}+a_{l}\right)^{2}}\right)\right],
\end{aligned}
$$

$$
\begin{aligned}
& f_{i 2}(\mathbf{x})=\sum_{i=1}^{n} \sum_{u=1}^{s_{i}+1} \rho_{i_{M_{i}[u]}}\left[\left(1+\sum_{m=1}^{4} \frac{\alpha_{i m}}{W_{i m}(\mathbf{x})}\right)\left(y_{i}-l y_{M_{i}[u]}\right)\right. \\
& +\left(\sum_{i=1}^{s_{i}^{*}} \frac{\xi_{i v}}{H_{i v}(\mathbf{x})}+\sum_{l=1}^{q} \frac{\gamma_{i l}}{F O_{i l}(\mathbf{x})}+\sum_{p=1}^{2} \frac{\kappa_{i p}}{U_{i p}}\right)\left(y_{i}-l y_{M_{i}[u]}\right) \\
& +\left(\sum_{\substack{j=1 \\
j \neq i}}^{n} F_{j_{M_{i}}[u]}(\mathbf{x})\left(\frac{\beta_{i l}}{M O_{i j}}+\frac{\beta_{i l}}{M O_{j i}}\right)\right)\left(y_{i}-l y_{M_{i}[u]}\right) \\
& -\sum_{\substack{j=1 \\
j \neq i}}^{n} F_{i M_{i}[u]}(\mathbf{x}) F_{j M_{i}[u]}(\mathbf{x})\left(\frac{\beta_{i j}\left(y_{i}-y_{j}\right)}{M O_{i j}^{2}}-\frac{\beta_{j i}\left(y_{j}-y_{i}\right)}{M O_{j i}^{2}}\right) \\
& -F_{i M_{i}[u]}\left(\frac{\alpha_{i 3}}{W_{i 3}^{2}}-\frac{\alpha_{i 4}}{W_{i 4}^{2}}+\sum_{v=1}^{s_{i}^{*}} \frac{\xi_{i v}}{H_{i v}^{2}(\mathbf{x})}\left(y_{i}-l y_{M^{*}{ }_{i}[v]}\right)\right) \\
& +\left(F_{i M_{i}[u]} \sum_{l=1}^{q} \frac{\gamma_{i l} \sin \psi_{l}\left(\left(y_{i}-o_{l 2}\right) \cos \psi_{l}+\left(x_{i}-o_{l 1}\right) \sin \psi_{l}\right)}{F O_{i l}^{2}(\mathbf{x})\left(r_{i v}+b_{l}\right)^{2}}\right) \\
& \left.-\left(F_{i M_{i}[u]} \sum_{l=1}^{q} \frac{\gamma_{i l} \cos \psi_{l}\left(\left(x_{i}-o_{l 1}\right) \cos \psi_{l}+\left(y_{i}-o_{l 2}\right) \sin \psi_{l}\right)}{F O_{i l}^{2}(\mathbf{x})\left(r_{v}+a_{l}\right)^{2}}\right)\right] \\
& f_{i 3}=1+\sum_{u=1}^{s_{i}+1} \frac{F_{i M_{i}[u]} \kappa_{i 1}}{U_{i 1}^{2}} \text { and } \\
& f_{i 4}=1+\sum_{u=1}^{s_{i}+1} \frac{F_{i M_{i}[u]} \kappa_{i 2}}{U_{i 2}^{2}} .
\end{aligned}
$$

Introducing convergence parameters $\delta_{i 1}, \delta_{i 2}>0$, we define the acceleration-based controllers as:

$$
\left.\begin{array}{rl}
\sigma_{i 1} & =-\left(\delta_{i 1} v_{i}+f_{i 1} \cos \theta_{i}+f_{i 2} \sin \theta_{i}\right) / f_{i 3}, \\
\sigma_{i 2} & =-\left(\delta_{i 2} \omega_{i}+\frac{L}{2} f_{i 2} \cos \theta_{i}-\frac{L}{2} f_{i 1} \sin \theta_{i}\right) / f_{i 4} .
\end{array}\right\}
$$

\section{Stability ANALYSis}

The acceleration controllers $\sigma_{i 1}$ and $\sigma_{i 2}$ given by (10) above would vanish at the center of the target and hence the vehicle will come to a complete rest at the goal position. Thus $\mathbf{x}_{e}=\left(p_{11}, p_{12}, \theta_{1}, 0,0, p_{21}, p_{22}, \theta_{2}, 0,0, \ldots, p_{n 1}, p_{n 2}, \theta_{n}, 0,0\right)$ is an equilibrium point of system (1). Moreover, on the domain $D$
1) $L(\mathbf{x})>0 \forall \mathbf{x} \in D \backslash \mathbf{x}_{e}$ and $L\left(\mathbf{x}_{e}\right)=0$;
2) $\dot{L}(\mathbf{x}) \leq 0$ and $\dot{L}\left(\mathbf{x}_{e}\right)=0$;
3) $\dot{L}=-\delta_{i 1} v_{i}^{2}-\delta_{i 2} \omega_{i}^{2} \leq 0$.

This implies that $\mathbf{x}_{e}$ is a stable equilibrium point.

\section{SIMULATION}

In this section, we demonstrate the simulation results for the movements of the car-like robots in a constrained workspace cluttered with obstacles via the landmarks. We numerically verify stability of the system via the Lyapunov function. Values of the parameters used in the simulation are given in Table I. The fixed obstacles and landmarks were generated randomly.

In Fig. 4 and Fig. 5, we notice that the car-like robots starts from their initial positions, move towards their targets via selected landmarks that are in their field of view. The carlike robots simultaneously avoided any obstacles along their path. 
Proceedings of the Fourth International Conference on Inventive Systems and Control (ICISC 2020)

IEEE Xplore Part Number: CFP20J06-ART; ISBN: 978-1-7281-2813-9

TABLE I. VALUES OF PARAMETERS UTILIZED IN THE SIMULATIONS.

\begin{tabular}{|l|c|c|}
\hline & Figure 4 & Figure 5 \\
\hline Initial Positions (m) & $(10,15),(10,70)$ and & $(30,5),(10,4)$ \\
& $(70,70)$ & $(70,70)$ \\
\hline Workspace Dimension & $0 \leq z_{1} \leq 80$, & $0 \leq z_{1} \leq 60$, \\
& $0 \leq z_{2} \leq 80$ & $0 \leq z_{2} \leq 60$ \\
\hline Number of Landmarks & 20 & 15 \\
Number of Obstacles & 15 & 10 \\
Maximum radius of obstacles (m) & $a_{i}=3, b_{i}=3$ & $a_{i}=3, b_{i}=3$ \\
Field of view $(\mathrm{rad})$ & $\pi / 10$ & $\pi / 10$ \\
Initial velocities $(\mathrm{m} / \mathrm{s})$ & 5 & 5 \\
\hline Final Positions $(\mathrm{m})$ & $(70,30),(40,60)$ and & $(50,30),(30,12)$ \\
& $(10,30)$ & $(10,7)$ \\
\hline Convergence Parameters & $\delta_{i 1}=\delta_{i 2}=15$, & $\delta_{i 1}=\delta_{i 2}=15$, \\
& for $i=1,2,3$. & for $i=1,2,3$. \\
\hline Minimum distance $\mathrm{D}^{*}(\mathrm{~m})$ & 10 & 10 \\
\hline
\end{tabular}

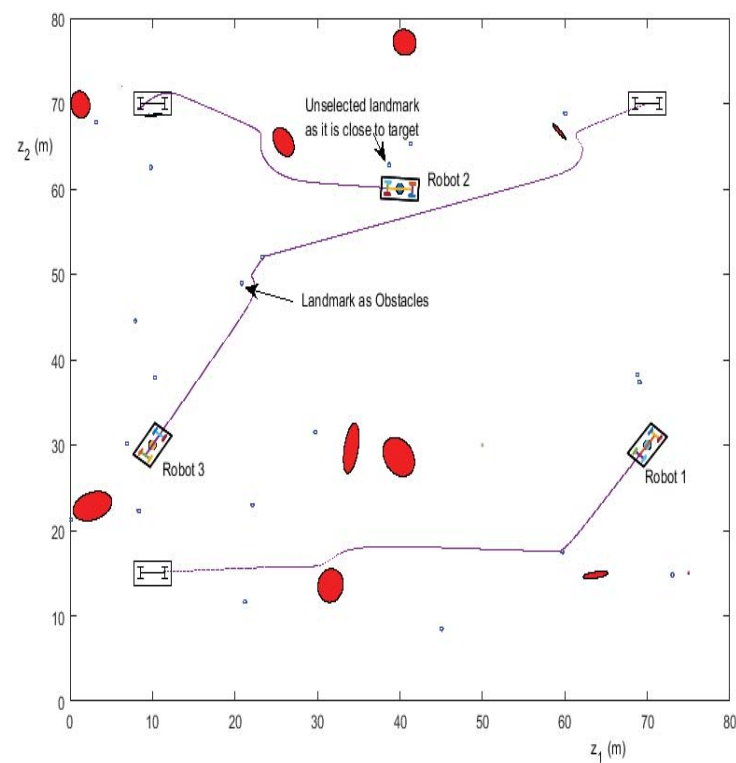

Fig. 4. Trajectory of the car-like mobile robots via selected landmarks.

\section{CONCLUSION}

This paper presents a new solution to the landmark navigation problem of multiple nonholonomic car-like robots which is governed by the Lyapunov-based control scheme. The landmarks are used as navigation-aids or waypoints to guide the planer robots to their targets in a bounded workspace which consists of fixed obstacles of random sizes and positions. The landmark attractions, obstacle avoidances, workspace and steering restrictions have been carefully incorporated into a Lyapunov function from which the control laws are extracted. Future work can possibly be the creation of new landmarks to improve navigation and also consider randomised roles of such navigation-aids.

\section{REFERENCES}

[1] K. Shojaei, "Neural adaptive robust output feedback control of wheeled mobile robots with saturating actuators," IET Control Theory and Applications, vol. 11, pp. 504-515, March 2017.

[2] B. Sharma, J. Vanualailai, and S. Singh, "Motion planning and posture control of multiple n-link doubly nonholonomic manipulators," Robotica, vol. 35, pp. 1-25, March 2015.

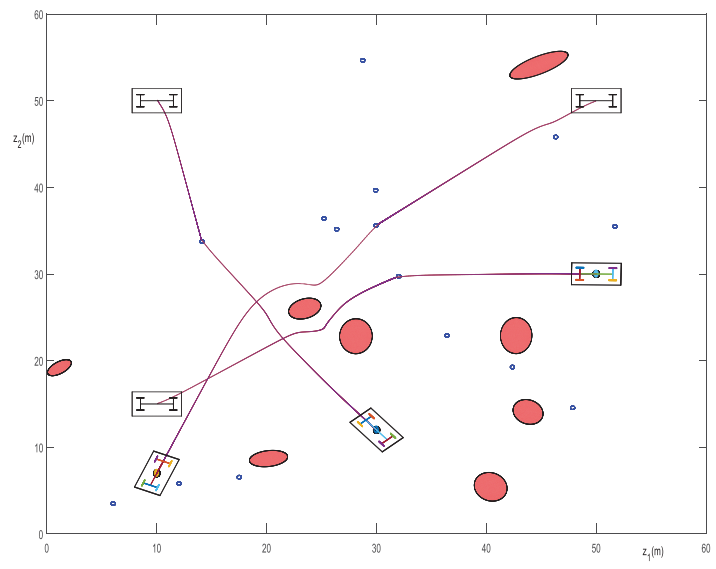

Fig. 5. Trajectory of the car-like robots via selected landmarks.

[3] B. Sharma, J. Raj, and J. Vanualailai, "Navigation of carlike robots in an extended dynamic environment with swarm avoidance." International Journal of Robust and Nonlinear Control, vol. 28, p. 678698, 2018

[4] K. Shojaei, "Neural adaptive output feedback formation control of type (m, s) wheeled mobile robots," Int. J. Adapt. Control Signal Process, vol. 29, p. 855876, 2015.

[5] X. Xing, R. Zhou, and L. Yang, "The current status of development of pedestrian autonomous navigation technology," in Proceedings of the 26th Saint Petersburg International Conference on Integrated Navigation Systems (ICINS), May 2019.

[6] M. Ataei and A. Yousefi-Koma, "Three-dimensional optimal path planning for waypoint guidance of an autonomous underwater vehicle," Robotics and Autonomous Systems, vol. 67, pp. 23 - 32, 2015, advances in Autonomous Underwater Robotics.

[7] Y. Yingmin and H. Xiangru, "Robot simultaneous localization and mapping based on self-detected waypoint," Cybernetics and Information Technologies, vol. 16, pp. 212-221, 2016.

[8] K. S. Gunther, K. Axram, and T. Duckett, "Physical analogies for rapid robot motion planning and execution in dynamic environments," Transactions of the IMACS/SICE international Symposium on Robotics, Mechatronics and Manufacturing Systems, pp. 3-8, 1993.

[9] H. Jagannathan, "Landmark-based robot navigation enhanced with color interest operators," in Proceedings of the Society of Photo-Optical Instrumentation Engineers (SPIE), vol. 3959, 2000, pp. 480-489.

[10] A. Dawadee, J. Chahl, and N. Nandagopal, "A method for autonomous navigation of uavs using landmarks," in Proceedings of the 16th Australian Aerospace Congress, 022015.

[11] A. Bais and R. Sablatnig, "Landmark based global self-localization of mobile soccer robots," in Lecture Notes in Computer Science, Hydrabad, India, January 2006, pp. 842-851.

[12] H. Fujii, Y. Ando, T. Yoshimi, and M. Mizukawa, "Shape recognition of metallic landmark and its application to self-position estimation for mobile robot," Journal of Robotics and Mechatronics, vol. 22, no. 6 , pp. 718-725, 2010.

[13] H. Hu and D. Gu, "Landmark-based navigation of mobile robots in manufacturing," in 7th IEE International Conference on Emerging Technologies and factory Automation Proceedings, October 1999, pp. 121-128. 
Proceedings of the Fourth International Conference on Inventive Systems and Control (ICISC 2020) IEEE Xplore Part Number: CFP20J06-ART; ISBN: 978-1-7281-2813-9

[14] P. Boucher, "Waypoints guidance of differential-drive mobile robots with kinematic and precision constraints," Robotica, vol. 32, pp. 1 24, 072014

[15] J. Borenstein, H. R. Everett, L. Feng, and D. Wehe, "Mobile robot positioning - sensors and techniques," Invited paper for the Journal of Robotic Systems, Special Issue on Mobile Robots, vol. 14, no. 4, pp. 231-241, 1997.

[16] B. Sharma, J. Vanualailai, and S. Singh, "Tunnel passing maneuvers of prescribed formations," International Journal of Robust and Nonlinear Control, vol. 24, no. 5, pp. 876-901, 2014.

[17] A. Prasad, B. Sharma, and J. Vanualailai, "A solution to the motion planning and control problem of a car-like robot via a single layer perceptron," Robotica, vol. 32, no. 6, pp. 935-952, 2014.

[18] L. Frommberger, "Representing and selecting landmarks in autonomous learning of robot navigation," Springer), vol. 5314, pp. 488-497, 2008. 